\title{
Aggregate simulation modeling with application to setting the CONWIP limit in a HMLV manufacturing cell
}

\author{
Shahriar Khan ${ }^{a}$ and Charles Standridge ${ }^{b *}$
}

${ }^{a}$ Grand Rapids Chair Company, 1250 84th St SW, Byron Center, MI 49315 USA

${ }^{b}$ Grand Valley State University, 301 Fulton Avenue West, Grand Rapids, MI 49504 USA

\begin{tabular}{l}
\hline C H R O N I C L E \\
\hline Article history: \\
Received July 182018 \\
Received in Revised Format \\
July 252018 \\
Accepted October 292018 \\
Available online \\
October 292018 \\
\hline Keywords: \\
Aggregate simulation modeling \\
High-Mix \\
Low-Volume Manufacturing \\
CONWIP \\
Production Flow \\
Discrete-Event Simulation \\
\hline
\end{tabular}
A B S T R A C T

Concepts for aggregate modeling and simulation are presented and applied for the setting of the parameters of a CONWIP flow control system within the steel products cell at Grand Rapids Chair, a high-mix, low-volume manufacturing environment. Aggregation was accomplished by combining the over 100 types of products into one. This resulted in a single arrival process, routing modeled as random movement through workstations, and a single operation time distribution for each production operation. Aggregation reduced the effort required for model development, data analysis, validation, and simulation. The CONWIP control was shown to be effective in managing the work-in-process. A CONWIP parameter of 50 resulted in an equivalent throughput to that achieved with no limit on work-in-process. Lead time can be reduced by approximately $20 \%$ by increasing the CONWIP parameter to 58 .

Production Flow

(C) 2019 by the authors; licensee Growing Science, Canada

\section{Introduction}

The use of discrete-event simulation modeling in the analysis of manufacturing systems is well established. An important question in developing a model of such a system is: What level of detail should be used? Etman et al. (2011) argue that including all the details of a complex system, such as a High-Mix, Low-Volume (HMLV) production system, in a simulation model makes this approach impractical due to lengthy development time and large computational requirements. They provide a specific application of aggregate modeling as a way of overcoming these challenges. In this work, a brief general discussion of aggregate modeling concepts is provided along with an illustrative application for setting the parameter of a flow control system in HMLV production. The general discussion provides a way to think about aggregate model building. The application shows the utility and effectiveness of this approach. In addition, utility of the integration of lean thinking and tools to support aggregate model development is demonstrated. The use of order data and observed cycle time data to estimate model parameters is shown.

* Corresponding author

E-mail: skhan@grandrapidschair.com (C. Standridge)

2019 Growing Science Ltd.

doi: $10.5267 / j . j$ ijiec.2018.10.002 
Generally, aggregate modeling has to do with combining some elements of the system such that fewer details are represented, making it easier and less time consuming to develop the model as well as reducing the computational requirements for simulation, without affecting the utility of the modeling and simulation activity in addressing the system issues at hand. This implies that the aggregation should not change the estimated values of the performance measures resulting from the simulation. How aggregation is accomplished must be specific to each model. For example, Hopp and Spearman (2011) develop the idea of effective processing time at a workstation. Using this technique the average and standard deviation of the processing time at the workstation are computed to include any detractors such as down time, batching, and rework. Etman et al. (2011) apply this idea to semiconductor production. Braglia et al. (2011) apply the same idea for setting the CONWIP limit through identifying a bottleneck work area.

The concepts of aggregate modeling can be applied to HMLV production systems. Such a system can produce many products, perhaps over 100, with each having a unique route through a set of work areas, perhaps 5-30. In such a situation, all products are produced to order, so the lead time from order receipt to completion of production is of interest. Estimating lead time using a detailed simulation model would require modeling the following.

- The production route of each product,

- One arrival process for each product, including estimating the parameters,

- The operation time distribution for each product and work area combination, including estimating the parameters.

Such analyses would be time consuming and perhaps for products with few orders would be difficult to do. In addition, model validation would include examining the movement of each product through the system, a time consuming activity.

Thus, the aggregate modeling approach can be very helpful. Aggregation can be accomplished by consolidating all products into one product. Thus, only one arrival process is needed. The route of each product can be selected at random using the probability of any product moving from its current work area to any of the following work areas. The cycle time at each work area is modeled as a single distribution, which does not depend on the product. Therefore, the aggregate model would require less development time, less detailed data, less data analysis, and less effort in validation than the detailed model.

These concepts for aggregate modeling are applied to the steel products cell at Grand Rapids Chair Company (GR Chair), a made-to-order furniture manufacturer of chairs and tables for commercial foodservice spaces. GR Chair sells hundreds of different products and processes thousands of orders per year. The GR Chair manufacturing plant has primary work cells: steel products, wood products, and tables. The steel cell, which produces chairs, chair frames, table bases and small parts, is of interest. This cell manufactures over 100 different products. In 2015, approximately 14,500 orders for these products were processed, $95 \%$ of which were delivered on time. However, only $54 \%$ of orders were manufactured on time. On time delivery was achieved by keeping a gap between the delivery date and the manufactured required date of at least 2 working days, which means that GR Chair is unnecessarily holding up to 2 days of finished goods inventory. Thus, improving flow control in the steel cell to reduce the need for finished goods inventory is a worthwhile objective.

One simple way to control flow is to establish an upper bound on the work-in-process (WIP) inventory. This can be accomplished by using a near constant WIP system, or CONWIP system (Spearman, et al. 1990). The only parameter of a CONWIP system is the pre-established maximum WIP level that will be referred to as the CONWIP limit. The ideal CONWIP limit is the smallest value that does not constrain throughput and equivalently, as shown by Little's Law (Little, 1961), minimizes lead time. If the mix of jobs changes, which is highly likely in an HMLV environment, the CONWIP system dynamically adapts 
since it has only one parameter. Discrete event simulation can be employed to establish the relationship between the CONWIP limit and lead time and thus help in setting the CONWIP limit.

Aggregate simulation model development is supported by the construction of a value stream map (VSM) from which the aggregate simulation model can be derived. A VSM approach for HMLV manufacturers with application is described by Matt (2014). The introduction of synchronization points in front of merge-activities in the value stream, and the splitting of customer orders into suitable production orders and equal time increments of work are the major contributions of this effort.

The value stream mapping processes alone is not sufficient for establishing the value of the CONWIP parameter. As was pointed out by Marvel and Standridge (2009), a lean transformation process based on a VSM does not typically validate the future state (e.g. establish the value of a flow control parameter) before implementation. Thus, there is no guarantee that a lean transformation will meet measurable performance objectives (e.g. a lead time target). Marvel and Standridge proposed using modeling and simulation to address this lean shortcoming and describe a process for so doing. Simulation results quantify lean manufacturing's impact regarding resource requirements and performance (Detty \& Yingling 2000). Miller et al. (2010) present a case study that further emphasizes this point and shows the benefits of such a validation.

Maas and Standridge (2015) present an integrated lean and non-aggregate simulation model process supported by the analysis of order history information to reduce the lead time for an existing electronics assembly system. Four order processing sequencing alternatives for improving on-time delivery were evaluated and the best of the four identified based on the average and variability in the on-time delivery metric.

A different approach was found in Wright and Standridge (2016) for lead time reduction by transformation to cellular production. Quick Response Manufacturing (QRM) ideas (Suri, 2010) drive the transformation primarily by minimizing customer lead time. The case study shows that lead time can be reduced by following a cellular manufacturing approach in HMLV industries such as GR Chair.

Pergher and Vaccaro (2014) develop a combined modeling and simulation and Electre TRI process for setting a CONWIP level and illustrate the procedure with an industrial example. Modeling and simulation are used to compute performance measures such as throughput for various CONWIP levels and Electre TRI is used to select among them.

Romagnoli (2015) discusses using modeling and simulation to set the CONWIP level in a make-to-order production environment that is similar to GR Chair. However, aggregate modeling is not used other than to organize individual products into families. A VSM is developed to describe the system. Cycle times are modeled using normal distributions though our experience is that cycle times tend to be skewed to the right and thus gamma distributions may be more appropriate. The production strategy includes reordering jobs at each workstation rather than creating smooth flow as at GR Chair where sequencing of orders is done only before production is begun.

There are few studies comparing flow control systems in HMLV applications. Frazee and Standridge (2016) studied and compared two flow control strategies: CONWIP and POLCA (Suri, 2018). POLCA is a technique for controlling production flow between two work cells. Simply, cell capacity is limited to a fixed number of time units such as an hour. Before a production job can start in the first cell, there must be sufficient production capacity in both cells to process the job. They employed a non-aggregate model and simulation, concluding that CONWIP control performs better than the POLCA control for the HMLV system studied. The CONWIP control achieves maximum throughput at a lower maximum WIP level than POLCA. However, any issues with WIP gathering in one area of the system, which POLCA 
could prevent but CONWIP cannot, were not encountered in this system due to batch operations and relatively low utilizations of the manual operations.

A short procedure for aggregate modeling is presented, including the use of value stream mapping and data in the production data management system. A simulation-based experimental procedure for setting the CONWIP level is described. These ideas are applied to setting the CONWIP level for the steel value stream at GR Chair.

\section{Methods}

The steps of an aggregate modeling process are presented.

\subsection{Develop a value stream map}

A VSM is a lean management technique used to portray the flow of materials and information to create a product or service. A VSM generally includes the material and information flows for components and sub-assemblies. It also describes manufacturing, supplier and distribution information.

Thus, pre-requisite to the construction of the simulation model is the development of a VSM to document and analyze the flow of information and materials required to produce a product. Each work area and buffer area as well as information flows should be included in the VSM.

\subsection{Develop an aggregate simulation model}

The possibilities for and potential benefits of an aggregate simulation model of a HMLV production system were discussed previously.

A CONWIP control fits well with an aggregate modeling approach as it has only one parameter, the CONWIP limit, which is not dependent on the number of different products. Thus, aggregating all products into one is possible.

Kingman's equation or the VUT equation (1961) can be used to gain insight into how this aggregation might affect lead time estimation.

$$
\mathrm{LTq}=\mathrm{V} \times \mathrm{U} \times \mathrm{T}
$$

The VUT equation estimates the average waiting time in the buffer of a station $\left(\mathrm{LT}_{\mathrm{q}}\right)$. The $T$ term is the average cycle time at the station overall job types, which is not impacted by aggregation. The $U$ time is a function of the utilization of the station or percent non-idle time of the station. Like the $\mathrm{T}$ term, the utilization is not impacted by aggregation. $V$ is the variance time that depends on the variance of the cycle time of the station and the arrival process to the station. The former term is not impacted by aggregation as the cycle time probability distribution overall products is not changed. The latter term may increase slightly due to the aggregation. If the routing is random as opposed to a function of the product, the variance of the arrival process to each station may increase. Some routes may be generated

in the model that do not correspond to the route of any product. Thus, the estimate of the average waiting time at a station may be biased high and the results of the simulation of the aggregate model are best interpreted as an upper bound on the lead time.

Given the understanding gained from the VUT equation, aggregating all products into one seems reasonable. 


\subsection{Data Acquisition and Analysis}

Data concerning the number of orders processed per year, the number of products per order, the route of each order during production and the observed cycle time for each product at each work area are required. This information should be available in a production data management system.

The model requires the time between the arrival of orders. The production data management system will contain the number of orders in a year. Thus, the average time between orders can be computed.

The distribution of the time between orders is also required. Hopp and Spearman (2011) define the practical worst case as represented by a coefficient of variation $(\mathrm{Cv})$ of 1 . Thus, a conservative assumption would be that the time between arrivals distribution has a $\mathrm{CV}$ of 1 . This assumption requires the use of an exponential distribution since the mean and standard deviation of all exponential distributions are equal.

For each work area, the fraction of product going to any subsequent work area can be computed straightforwardly. Thus, the subsequent work area for a product completing an operation can be selected randomly.

Histograms of the number of products per order and the cycle time for each work area are generated. These can be used directly in the simulation model as opposed to fitting distribution functions to the data. For the number of products per order, this means using a probability mass function. The histogram of the cycle time combines times over different products. There is likely a different distribution of cycle time for each product. Thus, a non-homogenous data set results. Therefore, a good fit of single distribution function to the data seems unlikely.

\subsection{Design and Conduct the Simulation Experiment}

Table 1 shows a template for designing a simulation experiment.

Table 1

Design Template for a Simulation Experiment

\begin{tabular}{ll}
\hline Element of the Experiment & Values for a Particular Experiment \\
\hline Model Parameters & \\
Performance Measures & \\
Number of Replicates & \\
Simulation End Time & \\
\hline
\end{tabular}

The simulation estimates the values of the performance measures based on the values of the model parameters. Model parameters values are changed in a systematic way and the effect on the performance measure values observed. The number of replicates of the model is specified such that the confidence intervals estimated for the performance measures are not too wide. In our experience, 20 replicates is generally sufficient. The simulation end time is generally the end of a corporate planning period such as a month, a quarter, or a year.

\subsection{Validate the Model}

No model completely captures all the facets, details, and subtleties of what is being modeled, particularly an aggregate model. As was discussed previously, this is not necessarily bad as models that are "right" would be very costly and time consuming to build and use. 
However, this perspective also leads to a basic question: Is the model useful for making decisions and drawing conclusions about improving what is being modeled and simulated? The answer is Yes provided that validation of the model has been properly conducted.

As described by many authors, primarily Banks, Carson, Nelson, and Nicol (2009), Sargent (2013) and Law (2014), validation requires gathering evidence that the model and its computer implementation accurately represent the system with respect to the issues to be addressed and solution objectives. Validation is a matter of degree. As more evidence is obtained, the greater the degree of confidence that the model is valid increases. However, absolute confidence $(100 \%)$ cannot be achieved. There will always be some doubt as to whether a model is validated. Thus, validation has to do with comparing the system and the data extracted from it to a simulation model and experimental results.

What validation evidence to obtain and how to obtain it is model specific and requires knowledge of how the model is going to be used. Utilization, the percent of time parts are processed at a work area, is a useful quantity for validation of an aggregate model as seen in the discussion of the VUT equation above. The utilization computed from data in the production information system is compared to the utilization estimated by the simulation. A confidence interval for the latter is computed. If this interval contains the value of the utilization computed from the production information system data, then validation evidence is obtained.

Often, an HMLV production system modeled by an aggregate model has many work areas. Thus, a word of caution is in order about the impact of using multiple confidence intervals in validation. Bonferroni's inequality, as discussed in DeVore (2015), is shown in Eq. (2).

$$
\mathrm{P}(\text { all confidence intervals cover the actual value })>=1-\sum_{j}^{k} \alpha_{j} \text {. }
$$

Suppose the HMLV production system had 10 work areas and $95 \%$ confidence intervals $(\alpha=5 \%)$ for the utilization were computed. Thus, the probability that the utilization computed from the production information system was contained in all 10 confidence intervals may be as low as $50 \%$.

\subsection{Examine the Simulation Results and Draw Conclusions}

The performance measure values generated for each set of model parameters are examined and conclusions drawn. How this is done is specific to each modeling and simulation project. In general, results are presented using tables and graphs with confidence intervals showing the plausible range for values of interest.

\section{Results}

The results obtained by applying the aggregate modeling process to the steel cell at GR Chair are presented.

\subsection{Develop a value stream map}

The current state value stream map for the steel cell at GR Chair is shown in Fig. 1. The VSM shows that the Steel Cell is organized like a Quick Response Manufacturing (QRM) cell (Suri 2010). The flow is from the left to the right. However, not all jobs are processed at all stations as indicated by the percent value following "Job" in each station block. The number of workers and/or machines at each station follows the word "Resource". What operations a job needs depends on which of the over one hundred types the job is. The operation time per piece at each station depends on the job type as well. The number of pieces per job varies widely from 1 to 2280, with the majority of jobs consisting of no more than 50 pieces. 
The first workstation is fabrication that consists of three operations, which if needed by a job, are performed in parallel. These are bending, laser, and machining. When all three are completed, the job waits for an available WIP cart. Notice that the WIP carts form a natural CONWIP control.

There are four welding stations each supporting a different type of welding. Some jobs require welding. Those that do generally require one form of welding but some require more than one type of welding. The final three stations are metal finishing, paint, and assembly. These are performed in sequence for a job that needs any or all of them. Finished jobs are stored in the supermarket. Some jobs have wood parts such as wooden backs on steel framed chairs. The wood parts are processed by the wood cell in parallel with the steel parts. The wood and steel parts are assembled by the assembly operation.

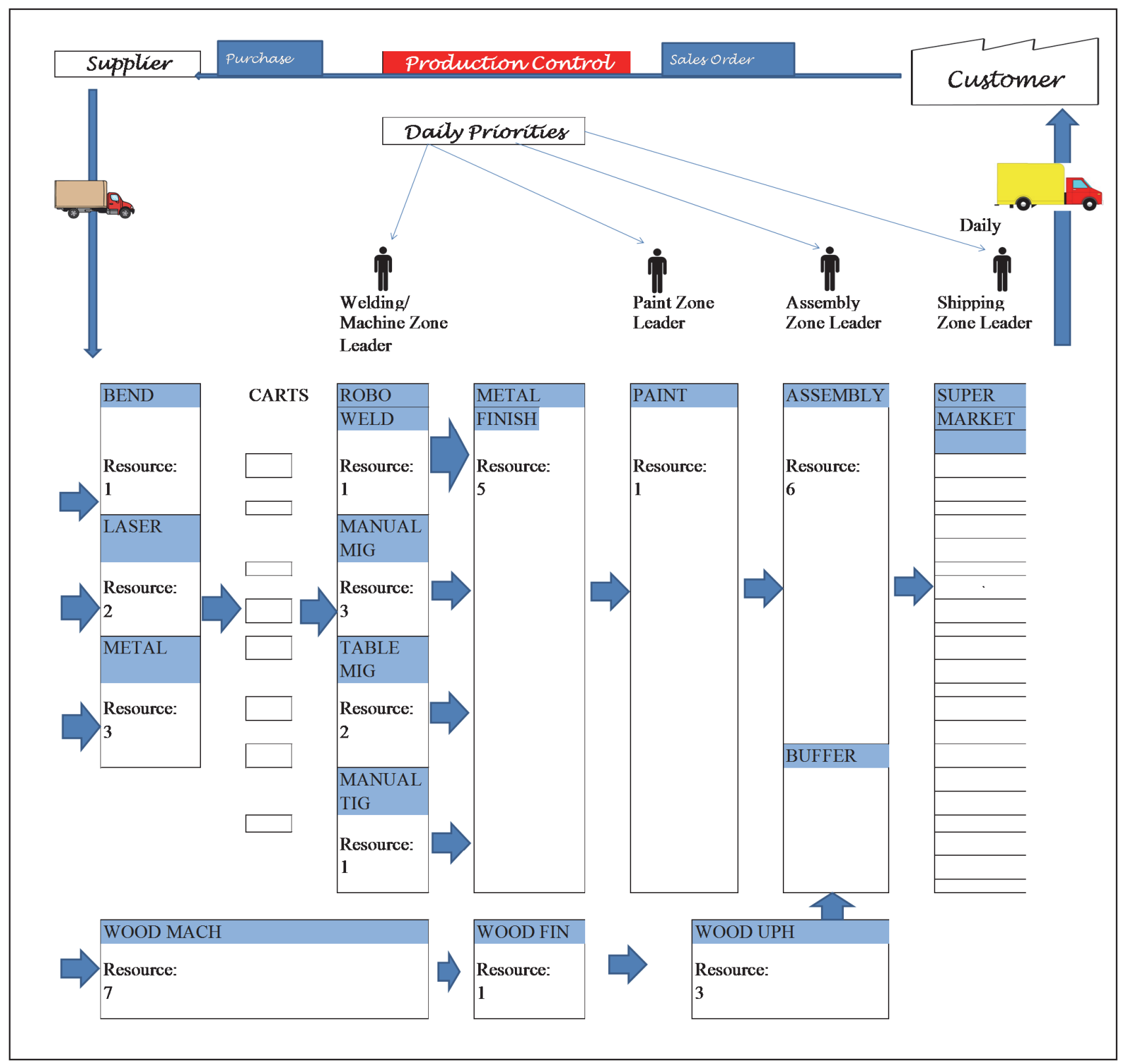

Fig. 1. GR Chair steel cell current state VSM 


\subsection{Develop an aggregate simulation model}

Aggregation can be accomplished by consolidating all 100 plus job types into one job type. Thus, only one arrival process is needed. The route of each job is selected at random using the probability of a job moving from its current station to any of the following stations. For example, when a job arrives, the probability that it requires the Bend station is $15 \%$, the probability that it requires the Laser station is $24 \%$, and the probability that it requires the Machining station is $49 \%$. After processing at all three stations is completed, the job waits for a WIP cart. The cycle time at a station is modeled as a single distribution using a histogram of the cycle time data collected from the production data management system.

\subsection{Data Acquisition and Analysis}

The EPICOR business data management software was used throughout the GR Chair plant to capture all production information. The order and production information from November 1, 2014 to October 31, 2015 consisted of more than 14,500 jobs. The information for each job included its type, the number of pieces, and the actual cycle time for each operation. An examination of the cycle time data revealed that some data points were infeasible due to either being too larger or too small. A set of rules was created using personal experience and expert opinion to establish a minimum and a maximum cycle time for each operation. It was assumed that any out of range data point was the result of a faulty manual input during cycle time entry. Such a point converted to the mode of the data set.

The corrected data set was used for the further analysis and the results prepared as input to the simulation model. There were cycle times corresponding to 95 different operations performed at the 13 workstations. In keeping with the aggregate modeling concept, these 95 operations were grouped by the work area at which each was performed, resulting in 13 aggregated operations. A cycle time histogram was generated for each of the 13 aggregated operations. These histograms were sampled to generate each cycle time in the simulation. The variation in the number of pieces per job was handled in the same way. A histogram was generated from the order data and a sample from the histogram generated during the simulation to set the number of pieces in an order. The histogram showing the distribution of the number of pieces in an order is shown in Fig. 2. A histogram showing the cycle time distribution for a single piece for the fabrication (machining) station is given in Fig. 3. This histogram is typical of those for the cycle time per piece at each of the workstations.

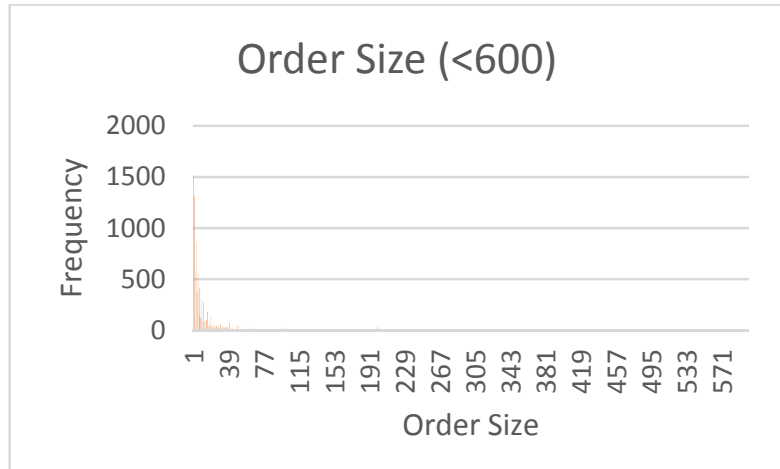

Fig. 2. Histogram of the Number of Pieces per Order

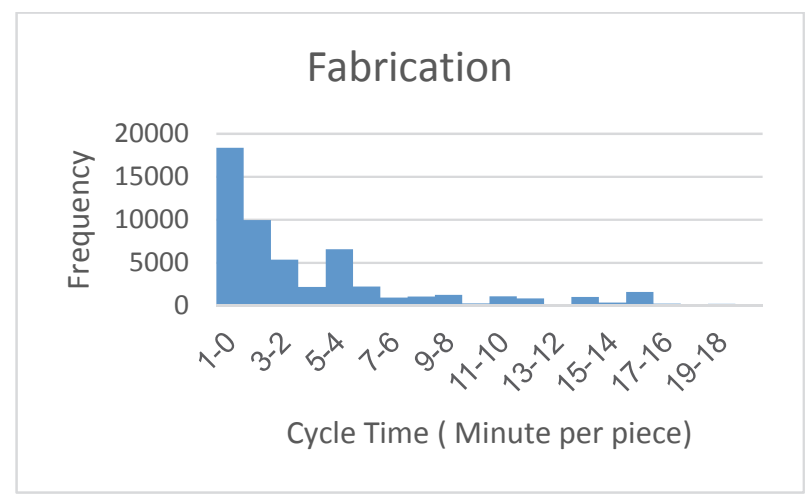

Fig. 3. Histogram of the Cycle Time at the Machining (Fabrication) Station

\subsection{Design and Conduct the Simulation Experiment}

The purpose of the simulation model was to help create and assess an effective CONWIP flow control mechanism for the steel cell. Thus, experimentation with the simulation model was needed to set the 
single CONWIP parameter, the CONWIP limit. If the CONWIP limit is set too high, then there is too much work-in-process in the cell which is undesirable. If the CONWIP limit is too low, then throughput is unnecessarily constrained and lead time for orders is too long. The CONWIP control can be implemented either logically by limiting the number of jobs in the steel cell or physically by limiting the number of WIP carts. The former was chosen. This allows the CONWIP control to correspond to the number of jobs in the cell as well as to include the first cell (bend, form, machining). Furthermore, the problem of assigning jobs to WIP carts in designing and implementing the CONWIP control is avoided. Jobs with few pieces could share a single WIP cart while jobs with many pieces would need to use multiple WIP carts. The design of the simulation experiment is shown in Table 2.

Table 2

Design of the Simulation Experiment

\begin{tabular}{ll}
\hline Element of the Experiment & Values for a Particular Experiment \\
\hline Model Parameter & CONWIP limit \\
Performance Measures & Number of jobs completed (throughput) \\
& Lead time \\
& Work in process (WIP) \\
& Maximum parts on WIP carts \\
& Utilization of workstation (Percent non-idle time) \\
Number of Replicates & 20 \\
Simulation End Time & 1 year (252 working days) \\
\hline
\end{tabular}

The only model parameter is the CONWIP limit. The primary performance measures are the number of jobs completed and the lead time. The work in process level is bounded by the CONWIP limit. The maximum number of parts on WIP carts is useful for determining how many carts will be needed. The simulation estimate of the utilization can be compared for validation purposes to the utilization computed from the data collected in EPICOR. The simulation is run for one year of working days corresponding to the length of time for which data was available. The simulation experiment is replicated 20 times.

\subsection{Validate the Model}

For the first experiment, the CONWIP level is set to a very high number that eliminates the effect of the CONWIP control. The simulation results for throughput estimate the maximum throughput the steel cell can achieve. The utilization results should be equivalent to those computed from the data in production data management system. This comparison is shown in Table 3. The utilization was calculated from the data in the production data management system using equation 3.

$$
\text { Utilization }=(\text { Cycle Time } / \text { Part }) \times(\text { Average Throughput } / \text { Day }) \times(\text { Time Available } / \text { Day })
$$

Table 3

Utilization Calculation for Model Validation

\begin{tabular}{|c|c|c|c|c|c|}
\hline \multirow[b]{2}{*}{ Station } & \multicolumn{5}{|c|}{$\begin{array}{r}\text { Utilization (in Percent) } \\
\end{array}$} \\
\hline & Calculated & Simulation Result & Absolute Difference & 95\% CI Lower & 95\% CI Upper \\
\hline BEND & 78 & 77 & 1 & 75 & 78 \\
\hline LASER & 57 & 55 & 2 & 50 & 59 \\
\hline MACHINING & 68 & 64 & 4 & 61 & 67 \\
\hline ROBO WELD & 75 & 72 & 3 & 69 & 75 \\
\hline MIG WELD & 55 & 54 & 1 & 52 & 57 \\
\hline TABLE WELD & 40 & 38 & 2 & 36 & 42 \\
\hline TIG WELD & 49 & 55 & 6 & 49 & 61 \\
\hline METAL FINISH & 45 & 44 & 1 & 43 & 46 \\
\hline PAINT & 53 & 53 & 1 & 50 & 55 \\
\hline ASSEMBLY & 57 & 52 & 5 & 50 & 54 \\
\hline
\end{tabular}


All absolute differences are no more than 5\% except for the TIG Weld station for which the absolute difference is about $7 \%$. The utilization computed from the production data management system for every workstation falls within the $95 \%$ confidence interval computed from the simulation results except for the Assembly station and the Machining station. At GRChair, the number of assembly workers varies daily based on anticipated workload between 3 and 8 workers. In the simulation, the number of workers at the assembly station was fixed at 4 . Thus, some difference in utilization is expected. Applying the Bonferroni inequality to the 10 confidence intervals shown in Table 3 leads to the result that there is as up to a $50 \%$ probability that at least one confidence interval will not contain the mean calculated from the production data management system. Thus, the result for the machining station is not surprising.

Therefore, it was concluded that the model and simulation are valid.

\subsection{Examine the Simulation Results and Draw Conclusions}

To create a baseline against which the performance of the CONWIP control could be compared, the model was simulated first with an infinite CONWIP limit. In this case, 11007 jobs were completed in a year with an average lead time of 7.8 hours. Furthermore, the WIP ranged from 4 to 96 jobs. Table 4 shows the throughput and lead time for various CONWIP limits.

Table 4

Simulation Results for Throughput and Lead Time by CONWIP Limit

\begin{tabular}{ccccc}
\hline CONWIP Limit & Throughput & & \multicolumn{2}{c}{$\begin{array}{c}\text { Lead Time (Hours) } \\
\text { 95\% Confidence Interval }\end{array}$} \\
\hline & & Average & Lower Bound & Upper Bound \\
\hline 35 & 10743 & 38.6 & 27.6 & 49.6 \\
40 & 10920 & 22.5 & 15.4 & 29.5 \\
45 & 10978 & 15.5 & 11.0 & 20.0 \\
50 & 11000 & 12.3 & 9.1 & 15.5 \\
53 & 11006 & 11.1 & 8.4 & 13.8 \\
54 & 11008 & 10.8 & 8.2 & 13.3 \\
55 & 11008 & 10.5 & 8.0 & 12.9 \\
56 & 11008 & 10.2 & 7.9 & 12.6 \\
57 & 11009 & 9.9 & 7.7 & 12.2 \\
\hline
\end{tabular}

Note that the throughput with limited WIP becomes equivalent to the throughput with unlimited WIP for CONWIP limits of 50 or more. The $95 \%$ confidence interval for lead time first contains 7.8 hours, the lead time with unlimited WIP, at a CONWIP level of 57. The average WIP is one measure of how well the CONWIP control keeps the WIP level stable. This measure for each CONWIP limit is shown in Figure 4. Note for CONWIP limits between 50 and 60 the average WIP stays in a narrow range between 36 and 42. The range limits are the minimum and maximum values of the average over the replicates of the simulation experiment.

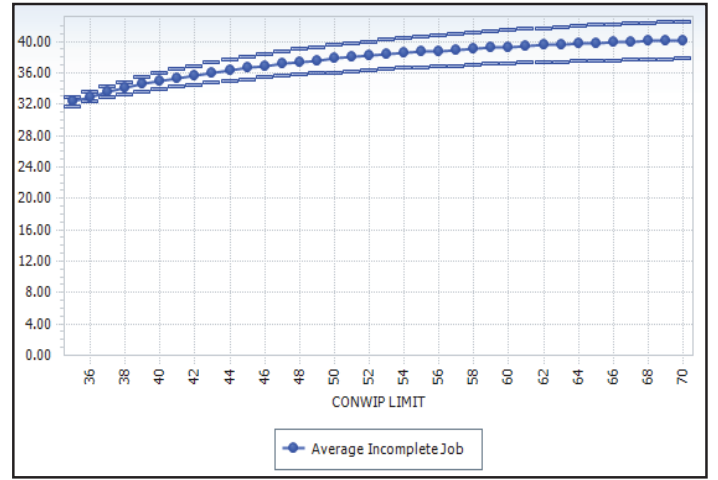

Fig 4. Average WIP by CONWIP limit

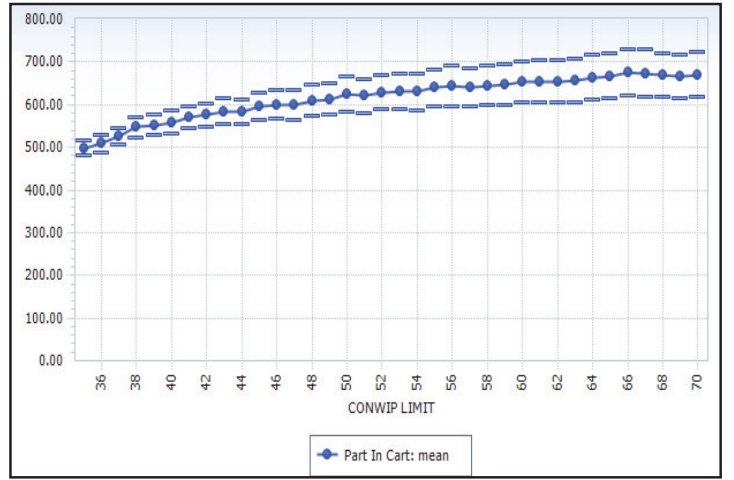

Fig 5. Average Number of Parts on Carts by CONWIP Limit 
Moving parts within the steel cell requires WIP carts. How many WIP carts to deploy is not straightforward to determine as part size varies widely. Figure 5 shows the average number of parts that need WIP carts for each CONWIP level. Note that for CONWIP levels greater than 50 the average number of parts on carts varies little between 625 and 650 . The range limits are the minimum and maximum values of the average over the replicates of the simulation experiment.

\section{Discussion}

The simulation results in Table 3 and Figure 4 show that the CONWIP control effectively manages the WIP. The maximum WIP is reduced from 96 to 50 with equivalent throughput per year achieved. The average WIP level is stable within a narrow range between 36 and 40 for a CONWIP level of 50, which supports some variation in the demand. If desired, the average lead time can be reduced by raising the CONWIP limit. For example, the average lead time can be reduced by about $20 \%$ by raising the CONWIP limit from 50 to 58. For CONWIP limits between 50 and 60, the average number of parts on carts ranges from about 625 to 650 as shown in Figure 5. The number of parts on any particular cart depends on part size as well as the mix of orders. Initially, the average number of parts per cart was estimated as 25 . If this is reasonable, then on average 25 carts would be in use. Five to ten additional carts could be made available to support periods of high demand and orders needing larger than average sized parts. It is feasible for GRChair to acquire and use 25 to 30 carts. More work is needed at GRChair to establish the number of carts actually needed.

\section{Conclusions}

The single major problem identified in GR Chair was the interruption in flow throughout the plant including the steel cell. This was seen in a number of ways.

1. Work area leaders requesting the completion of specific jobs from the upstream work areas

2. Time wasted re-scheduling jobs at work areas

3. Lack of synchronization between parallel work areas causing downtime.

4. Lack of standardization in processes causing unnecessary worker motion in machine setup and production

5. High finished goods inventory levels needed to ensure delivery on time

A combination of VSM, production data analysis, and aggregate modeling and simulation were used to help create flow by addressing items $1,2,3$, and 5. A CONWIP flow control mechanism was employed to ensure that neither too few nor too many jobs were in process in the steel cell. The CONWIP limit was set. A strict discipline of processing jobs at each workstation in the same sequence was established.

Aggregate modeling was shown to be effective. There is a single CONWIP flow control parameter that does not depend on the type of part or job. Thus, aggregating the various job types into one job type is possible. This significantly reduces the time needed to complete the analysis, including model building, data analysis, simulation and validation, without compromising the utility of the results. A CONWIP limit between 50 and 58 was shown to be effective in controlling work-in-process inventory while minimizing lead time.

\section{References}

Banks, J. A., Carson II, J. S., Nelson, B. L. \& Nicol, D. M. (2009). Discrete-Event systems simulation, 5th ed. New York : Pearson.

Braglia, M., Frosolini, M., Gabbrielli, R., \& Zammori, F. (2011). CONWIP card setting in a flow-shop system with a batch production machine. International Journal of Industrial Engineering Computations, 2(1), 1-18. 
Detty, R. B., \& Yingling, J. C. (2000). Quantifying benefits of conversion to lean manufacturing with discrete event simulation: a case study. International Journal of Production Research. 38(2), 429445.

Devore, J. L. (2015). Probability and statistics for engineering and the sciences, $9^{\text {th }}$. Boston: Cengage Learning.

Etman, L. F. P., Veeger, C. P. L., Lefeber, E., Adan, I. J., \& Rooda, J. E. (2011, December). Aggregate modeling of semiconductor equipment using effective process times. In Simulation Conference (WSC), Proceedings of the 2011 Winter (pp. 1790-1802). IEEE.

Hopp, W. J. \& Spearman, M. L. (2011). Factory physics: foundations of manufacturing management, $3^{\text {rd }}$ edition. Long Grove, IL : Waveland Press.

Kingman, J. F. C. (1961). The single server queue in heavy traffic. Mathematical Proceedings of the Cambridge Philosophical Society, 57(4), 902

Law, A. M. (2014). Simulation Modeling and Analysis, 5th ed. New York : McGraw-Hill.

Little, J. D. C. (1961). A Proof for the queuing formula: L = $\lambda$ W. Operations Research, 9(3), 383-387.

Maas, M., \& Standridge, C. R. (2015). Reducing lead times in a two-process cell using lean and simulation. Research Journal of Applied Sciences, Engineering, and Technology, 10(1), 15-21.

Matt, D. T. (2014). Adaptation of the value stream mapping approach to the design of lean engineer-toorder production systems: a case study. Journal of Manufacturing Technology Management, 25(3), 334-350.

Marvel, J. H. \& Standridge, C. R. (2009). A simulation enhanced lean design process. Journal of Industrial Engineering and Management, 2(1), 90-113.

Miller, G., Pawloski, J., \& Standridge, C.R. (2010). A case study of lean, sustainable manufacturing. Journal of Industrial Engineering and Management, 3(1), 11-32.

Pergher, I., \& Vaccaro, G. L. R. (2014). Work in process level definition: a method based on computer simulation and electre tri. Production, 24(3), 536-547.

Romagnoli, G. (2015). Design and simulation of CONWIP in the complex flexible job shop of a MakeTo-Order manufacturing firm. International Journal of Industrial Engineering Computations, 6(1), 117-134.

Sargent, R. G. (2013). Verification and validation of simulation models. Journal of simulation, 7(1), 1224.

Spearman, M.L., Woodruff, D.L., \& Hopp, W.J. (1990). CONWIP: A pull alternative to Kanban. International Journal of Production Research, 28(5), 879-894.

Suri, R. (2010). It's about time. New York: CRC Press.

Suri, R. (2018). The Practitioner's Guide to POLCA. New York: CRC Press.

Wright, D. \& Standridge, C. R. (2016). A case study of lead time reduction by transformation to cellular production. IOSR Journal of Engineering, 6(2), 53-58.

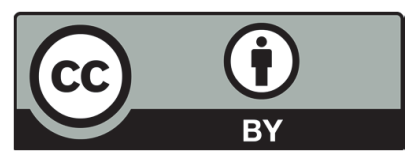

(C) 2019 by the authors; licensee Growing Science, Canada. This is an open access article distributed under the terms and conditions of the Creative Commons Attribution (CCBY) license (http://creativecommons.org/licenses/by/4.0/). 